\title{
Interpretation of the Superpave IDT Strength Test using a Viscoelastic-damage Constitutive Model
}

\author{
Ibrahim Onifade · Romain Balieu • Bjorn \\ Birgisson
}

Received: date / Accepted: date

\begin{abstract}
This paper presents a new interpretation for the Superpave IDT strength test based on a viscoelastic damage framework. The framework is based on continuum damage mechanics and the thermodynamics of irreversible processes with an anisotropic damage representation. The new approach introduces considerations for the viscoelastic effects and the damage accumulation that accompanies the fracture process in the interpretation of the Superpave IDT strength test for the identification of the Dissipated Creep Strain Energy (DCSE) limit from the test result. The viscoelastic model is implemented in a Finite Element Method (FEM) program for the simulation of the Superpave IDT strength test. The DCSE values obtained using the new approach is compared with the values obtained using the conventional approach to evaluate the validity of the assumptions made in the conventional interpretation of the test results. The result shows that the conventional approach over-estimates the DCSE value with increasing estimation error at higher deformation rates.
\end{abstract}

Keywords Asphalt Concrete - Dissipated Creep Strain Energy (DCSE) . Visco-Elastic-Damage · Anisotropic Damage · Superpave IDT strength test

\section{Introduction}

The SuperPave IDT test is a practical and reliable way to measure asphalt mixture tensile strength properties. The Superpave IDT test procedure consists of three different tests (resilient modulus test, creep test and strength test) which can be used to characterize mixture viscoelastic and strength properties. The Superpave IDT is used in the Mechanistic-Empirical Pavement Design Guide for the prediction of low temperature cracking of flexible pavements [Christensen and Bonaquist, 2004]. It has also been used for the evaluation of fatigue cracking and low-temperature

Ibrahim Onifade

Brinellvagen 23, Stockholm

Tel.: +46-764-096474

E-mail: onifade@kth.se 
cracking of asphalt mixtures by various researchers e.g., [Christensen and Bonaquist, 2004],

[Das et al., 2012b], [Kim and Wen, 2002], [Lytton et al., 1993].

In the Superpave IDT strength test, a disc-shaped sample of asphalt mixture is loaded diametrically until failure to obtain the tensile strength and fracture properties. The test is performed at a fast constant deformation rate to reduce the viscoelastic effect to simplify the interpretation of the test results. Compressive loads applied at opposite ends of the disc induce a fairly uniform tensile stress perpendicular to the direction of loading. Advantages of the Superpave IDT include that the test geometry can be easily obtained from cored field samples and the failure plane during the superpave IDT strength test is known a priori, hence failure limits can be accurately determined from measurements obtained on the failure plane. Another advantage of the superpave IDT strength test is that the point of macro-crack initiation can be identified based on the comparative evaluation of the changes in the vertical and horizontal deformation in the center region of the specimen as proposed by [Roque and Buttlar, 1992] and implemented in the AASHTO T322. The proposed procedure is usually used to obtain accurate strength parameters and fracture properties of the test specimen. One major disadvantage of the Superpave IDT test is that the test results are difficult to analyze due to the complex stress states that develops in the specimen.

Hot Mix Asphalt (HMA) Fracture Mechanics relies on the interpretation of the test results from the Superpave IDT strength test to identify the existence of fundamental crack growth thresholds below which only healable microdamage develops and above which non-healable crack initiation or growth occurs. The existence of these thresholds was identified by [Roque et al., 1999] in their work on the determination of crack growth rate parameters of asphalt mixtures using the Superpave IDT test. [Zhang et al., 2001b], [Zhang et al., 2001a] established that the Dissipated Creep Strain Energy (DCSE) and Fracture Energy (FE) density limits suitably define the lower and upper limit of the threshold respectively. The Fracture Energy is the area under the stress-strain curve up to the failure strain and the DCSE is deduced as the difference between the FE and the Elastic Energy (EE) [Roque et al., 2002b]. The EE is deduced using the resilient modulus obtained from the resilient modulus test.

The DCSE and FE limits have been used to simulate the crack growth in HMA by various researchers including [Roque et al., 2002a], [Zhang et al., 2001a], [Sangpetngam et al., 2003]. [Nguyen et al., 2013] proposed a fracture-based fatigue model where the DCSE and FE are used as parameters to characterize the fatigue behavior of different asphalt mixtures. [Roque et al., 2004] derived a parameter termed the "Energy Ratio" derived from the HMA Fracture Mechanics to predict top-down cracking performance of hot mix asphalt pavements. The Energy Ratio is defined as the ratio of the DCSE of the mixture obtained from the Superpave IDT strength test to the DCSE computed due to the applied loading. The DCSE threshold have also been incorporated in a framework for the prediction of fatigue cracking in asphalt pavements by [Dinegdae et al., 2015]. [Birgisson et al., 2007] proposed a strain energy-based fracture model combining an energy-based fracture criterion (accumulated DCSE) with a viscoelastic displacement discontinuity boundary element method to investigate crack growth in hot mix asphalt materials.

The major contributions of the HMA FM are the understanding of the discontinuous step-wise crack growth pattern of asphalt mixtures under fatigue loading 
and the establishment of thresholds between healable and non-healable damage [Zhang et al., 2001b], [Roque et al., 1999]. However, in the interpretation of the Superpave IDT using the HMA FM, there is no existing criterion for the development of micro-damage and no law that governs the evolution of the micro-damage to form macro-cracks. Interpretation of the Superpave IDT test results that provides the basis for the introduction of fundamental concepts required to accurately describe the characterization of materials for specification development, mixture design and pavement thickness design and performance evaluation should address the processes associated with micro crack initiation, evolution and macro-crack formation.

Recent work by [Onifade et al., 2015] led to the development of an energybased viscoelastic damage and fracture model which can be used to describe the process of micro-crack initiation, micro-crack propagation and subsequent macrocrack formation in viscoelastic materials. The model identifies the existence of a critical micro-crack threshold below which the material response is purely linearviscoelastic. The model characterizes the distributed damage or micro-crack in the material using an isotropic scalar damage parameter which is introduced into the constitutive equations to account for the damage effects on the overall material stiffness properties.

In this study, a new interpretation of the Superpave IDT strength test is proposed using the viscoelastic-damage model proposed by [Onifade et al., 2015] with further extension to consider anisotropic damage evolution due to the complex stress-state in the material during the Superpave IDT strength test. The damage model introduces energy potentials for the identification of an energy threshold for micro-crack initiation and its subsequent propagation. The new approach considers the effect of viscoelasticity and damage accumulation on the interpretation of the test result in the Superpave IDT strength test. The damage model is implemented in a Finite Element Method (FEM) program for the identification of the damage parameters and the simulation of the Superpave IDT strength test to predict the material response. The Superpave IDT resilient modulus and creep tests are used to obtain the viscoelastic material parameters and the deviation from the linear viscoelastic response is considered as damage in the Superpave IDT strength test. Furthermore, the test is carried at a low temperature $\left(10^{\circ} \mathrm{C}\right)$ and high deformation-rates to minimize the effects of plasticity on the test results.

\section{Conventional interpretation of test result from the Superpave IDT strength test.}

The Superpave IDT strength test is carried out to obtain the strength and fracture properties of mixtures. The strength properties are characterized using the observed stress and strain response at the center of the specimen. The horizontal tensile stress is obtained using Equation 1, where $P$ is the applied load, $t$ is the thickness of the test specimen and $d$ is the diameter of the test specimen. The horizontal tensile strain is obtained using the relationship in Equation 2, where $\triangle H$ is the horizontal deformation, and $G L$ is the gauge length. $C_{s x}$ and $C_{e x}$ are Correction factor introduced to correct for the effects of biaxial stress state and bulging on the test results. The correction factor $C_{s x}$ and $C_{e x}$ are functions of the Poissons 
ratio and the horizontal and vertical deformations, see [Roque and Buttlar, 1992] for details.

$$
\begin{gathered}
\sigma_{x x}=\frac{2 P \cdot C_{s x}}{\pi t d}, \\
\epsilon_{x x}=\frac{\triangle H \cdot C_{e x}}{G L},
\end{gathered}
$$

The HMA Fracture Mechanics (FM) identifies the existence of energy terms that correlate with the observed crack performance of asphalt mixtures. The energy terms include the Fracture Energy (FE) and the Dissipate Creep Strain Energy (DCSE) which is obtained as the difference between the FE and the Elastic Energy (EE). To simplify the analysis of the Superpave IDT strength test result, the EE is computed using the resilient modulus from the Superpave resilient modulus test. The FE, EE and DCSE can be obtained from the Superpave IDT strength test using Equation 3-5. Figure 1 shows a typical stress-strain response from the Superpave IDT strength test and the coventional approach to obtain the energy terms - DCSE and EE.

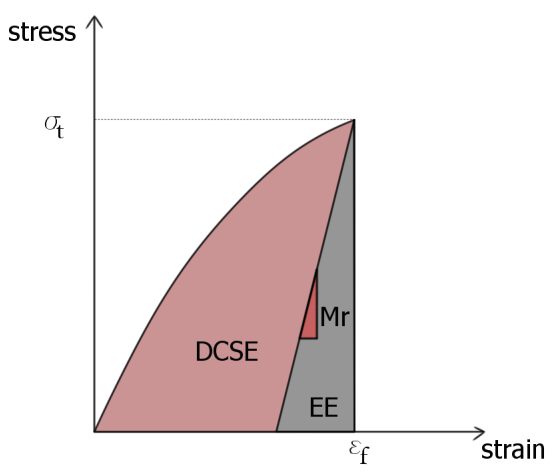

Fig. 1: Typical stress-strain response in the Superpave IDT strength test

$$
\begin{gathered}
F E=\int_{o}^{\epsilon_{f}} \sigma_{t} d \epsilon, \\
E E=\frac{\sigma_{t}^{2}}{2 \cdot M_{r}}, \\
D C S E=F E-E E,
\end{gathered}
$$

where, $\sigma_{t}$ is the tensile strength, $\epsilon_{f}$ is the failure strain and $M_{r}$ is the resilient modulus. 


\section{Shortcomings of the conventional approach in determining the DCSE and recommendations.}

The findings from the study by [Koh, 2009] highlights a major shortcoming in the present approach used to obtain the DCSE value. In [Koh, 2009], the fracture properties of open graded friction course materials are characterized using different test setups dog bone direct tension (DBDT) and the Superpave IDT test. Due to the shape of the test geometry and the mode of loading (direct tension) in DBDT, non-uniform stress distribution on the DBDT cross-section which results in higher local strain rate at the failure plane than the strain-rate observed in the Superpave IDT strength test.

Based on the strength test result from [Koh, 2009], the Fracture Energy (FE) and the resilient modulus $(M r)$ obtained using both DBDT and Superpave IDT strength test were similar for the same test specimens. However, the DCSE values obtained from both test setups were at variance. The DCSE values obtained from the Superpave IDT test are greater than the values obtained from the DBDT test. The test results from [Koh, 2009] suggests that the conventional method of interpretation of the Superpave IDT strength test results may result in an over-estimation of the DCSE value when the reduction in stiffness due to the accumulation of damage is not accounted for. This over-estimation can have a significant influence on response prediction of asphalt mixtures especially when the DCSE is used as a measure of resistance to damage or crack initiation.

The new approach recommends the consideration of the viscoelastic effects and the reduction in the material stiffness due to the damage (micro-crack) accumulation that accompanies the fracture process in determining the DCSE value. The following sections present an approach for the estimation of the damage accumulation during the strength test using a viscoelastic damage model.

\section{Viscoelastic Damage Model}

Recently [Onifade et al., 2015] proposed a new viscoelastic damage model based on energy balance with potentials for the identification of the critical threshold for micro-crack initiation and its consequent evolution based on thermodynamics of irreversible processes and Continuum Damage Mechanics.

The viscoelastic damage formulation presented in [Onifade et al., 2015] is based on the principle of strain equivalence and the damage represented using an isotropic scalar damage parameter. The generalized Maxwell model of n-terms with inclusion of a viscous strain $\left(\epsilon_{i}^{v}\right)$ from each of the dashpot components is used in the model formulation. The generalized Maxwell model is categorized into a long term equilibrium (time-independent) part with long term equilibrium stiffness $\left(E_{\infty}\right)$ and a series of non-equilibrium parts (time-dependent) with different stiffness $\left(E_{i}\right)$ and dashpot viscosity $\left(\eta_{i}\right)$. The total strain is additively decomposed into an elastic strain $\left(\epsilon_{i}^{e}\right)$ and a viscous strain $\left(\epsilon_{i}^{v}\right)$ on each Maxwell branch i.e. $\epsilon=\epsilon_{i}^{e}+\epsilon_{i}^{v}$.

The use of an isotropic scalar damage parameter for the material response prediction in the Superpave IDT strength test assumes similar strength characteristics in tension and compression, which is not a true representation of the behaviour of asphalt mixtures. This results in an over-estimation of the damage and equal stiffness reduction irregardless of the stress state at the different positions in the 
material. The stress states in the material during the Superpave IDT strength test is rather complex with a combination of compression, tension and shear stresses at different geometrical locations. For instance, in the Superpave IDT strength test, the regions close to the center of the specimen experience a compressive stress in the vertical direction and a tensile stress in the horizontal direction. The tensile stress in the horizontal direction results in the opening of micro-voids while the compressive stress in the vertical direction may result in the closing up of voids in the vertical direction. This results in the reduction of the material stiffness in the horizontal direction while the material retain its integrity in the vertical direction. The accumulated damage in the horizontal direction induces an anisotropic material behaviour under loading conditions with increase compliance in the principal damage direction. Therefore, the isotropic damage assumptions may not adequate for damage prediction in the Superpave IDT strength test. For this reasons, the proposed damage model in [Onifade et al., 2015] is further developed to consider anisotropic damage evolution and the energy equivalence principle is adopted to obtain symmetric stiffness and compliance tensors. However, the form of damage evolution law in [Onifade et al., 2015] is used in this paper.

The approach used for the formulation of anisotropic damage in [Voyiadjis et al., 2009], [Hansen and Schreyer, 1994], [Carol et al., 2001], [Abu Al-Rub and Voyiadjis, 2003], [Cordebois and Sidoroff, 1982], [Balieu and Kringos, 2015] based on the energy equivalence principle have been adopted and used for the further development of the viscoelastic damage model. The isotropic scalar damage parameter $D$ is replaced by a symmetric second-order tensor (D) and the integrity tensor $\phi$ is identified and expressed as $\phi=\boldsymbol{I}-\mathbf{D}$, where $\boldsymbol{I}$ is a second-order identity matrix and principal values of both $\phi$ and $\mathbf{D}$ varies from 0 to 1 .

Considering the energy equivalence principle, the Helmholtz free energy for the long term and equilibrium part of the Generalized Maxwell model can be written as:

$$
\psi=\frac{1}{2} \cdot\left[\epsilon: M^{-1}: \overline{E_{\infty}}: M^{-T}: \epsilon+\sum_{i}^{n}\left(\epsilon-\epsilon_{i}^{\boldsymbol{v}}\right): M^{-1}: \overline{\boldsymbol{E}}_{i}: M^{-\boldsymbol{T}}:\left(\epsilon-\epsilon_{i}^{\boldsymbol{v}}\right)\right]
$$

where $\overline{\boldsymbol{E}_{\infty}}$ and $\overline{\boldsymbol{E}}_{\boldsymbol{i}}$ are the effective long term and equilibrium stiffness of the $i^{\text {th }}$ term of the Generalized Maxwell model, and $\boldsymbol{M}$ is a fourth-order damage tensor. The derivative of the free energy with respect to the strain $\epsilon$ gives the relationship between the nominal stress $\boldsymbol{\sigma}$ and the effective stress $\overline{\boldsymbol{\sigma}}$ :

$$
\bar{\sigma}=M: \sigma,
$$

The relationship between the effective strain and nominal strain is expressed in a similar manner as:

$$
\bar{\epsilon}=M^{-T}: \epsilon
$$

where the the fourth-order damage effect tensor $M$ is defined in terms of the integrity tensor $\phi$ (where $\phi=I-\mathbf{D}$ ) according to [Voyiadjis et al., 2009] as:

$$
\boldsymbol{M}=\frac{1}{2}\left(\boldsymbol{I} \otimes \boldsymbol{\phi}^{-1}+\boldsymbol{\phi}^{-1} \otimes \boldsymbol{I}\right)
$$


The matrix representation of $\boldsymbol{M}^{-\mathbf{1}}$ tensor exhibits the diagonal form shown in Equation 10 in the principal axis of damage. A similar representation of the fourthorder damage tensor was used by [Carol et al., 2001], [Abu Al-Rub and Voyiadjis, 2003]:

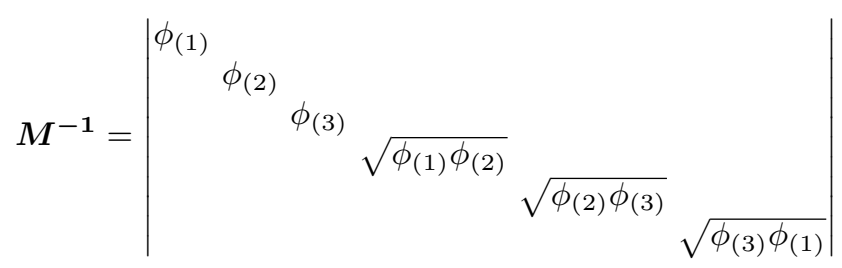

The relationship between the damaged stiffness and the undamaged (effective) stiffness for the long term and equilibrium parts can be established from the free energy in Equation 6.

$$
\begin{gathered}
E_{\infty}=M^{-1}: \bar{E}_{\infty}: M^{-T} \\
E_{i}=M^{-1}: \bar{E}_{i}: M^{-T}
\end{gathered}
$$

The long term stiffness and the equilibrium stiffness can be combined and written in terms of the instantaneous stiffness $\left(E_{o}\right)$ expressed as:

$$
E_{o}=M^{-1}: \bar{E}_{o}: M^{-T}
$$

where

$$
\overline{\boldsymbol{E}}_{\boldsymbol{o}}=\overline{\boldsymbol{E}}_{\infty}+\sum_{i}^{n} \overline{\boldsymbol{E}}_{\boldsymbol{i}}
$$

The effective instantaneous stiffness $\overline{\boldsymbol{E}}_{\boldsymbol{o}}$ can be written in terms of the effective Lame's constants $\bar{\lambda}_{o}$ and $\bar{G}_{o}$ and Equation 14 is expressed as:

$$
\overline{\boldsymbol{E}}_{\boldsymbol{o}}=\boldsymbol{M}^{-\mathbf{1}}: \boldsymbol{M}^{-\boldsymbol{T}}\left(\bar{\lambda}_{o}+2 \bar{G}_{o}\right)
$$

The matrix representation of the stiffness matrix in the principal damage direction is given as:

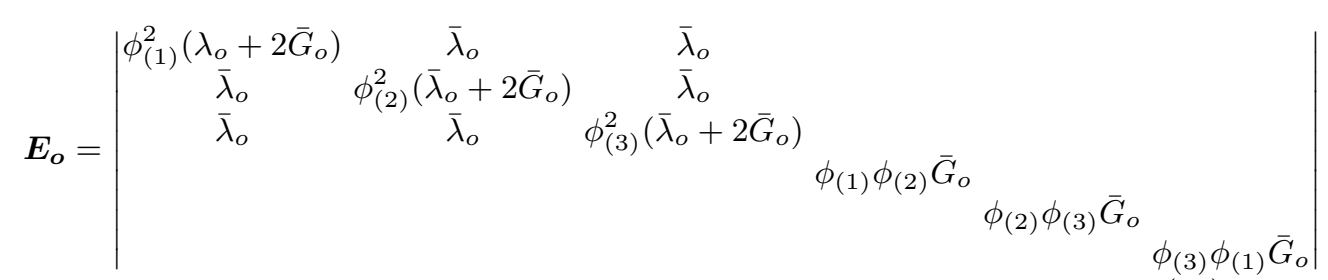

The thermodynamic dual $\boldsymbol{Y}$ of the damage variable $\boldsymbol{\phi}$ is obtained by differentiating the Helmholtz expression in Equation 6 by the damage variable to obtain:

$$
\begin{gathered}
\boldsymbol{Y}=-\frac{\partial \psi}{\partial \phi}=\frac{1}{2} \cdot\left[\boldsymbol{\epsilon}: \frac{\partial \boldsymbol{M}^{-1}}{\partial \phi}: \overline{\boldsymbol{E}_{\infty}}: \boldsymbol{M}^{-\boldsymbol{T}}: \boldsymbol{\epsilon}\right. \\
+\boldsymbol{\epsilon}: \boldsymbol{M}^{-1}: \overline{\boldsymbol{E}_{\infty}}: \frac{\partial \boldsymbol{M}^{-\boldsymbol{T}}}{\partial \boldsymbol{\phi}} \boldsymbol{\epsilon}
\end{gathered}
$$


$+\sum_{i}^{n}\left(\boldsymbol{\epsilon}-\boldsymbol{\epsilon}_{\boldsymbol{i}}^{\boldsymbol{v}}\right): \frac{\partial \boldsymbol{M}^{-1}}{\partial \phi}: \overline{\boldsymbol{E}}_{\boldsymbol{i}}: \boldsymbol{M}^{-\boldsymbol{T}}:\left(\boldsymbol{\epsilon}-\boldsymbol{\epsilon}_{\boldsymbol{i}}^{\boldsymbol{v}}\right)$

$\left.+\sum_{i}^{n}\left(\boldsymbol{\epsilon}-\boldsymbol{\epsilon}_{\boldsymbol{i}}^{\boldsymbol{v}}\right): \boldsymbol{M}^{-\boldsymbol{1}}: \overline{\boldsymbol{E}}_{\boldsymbol{i}}: \frac{\partial \boldsymbol{M}^{-\boldsymbol{T}}}{\partial \phi}\left(\boldsymbol{\epsilon}-\boldsymbol{\epsilon}_{\boldsymbol{i}}^{\boldsymbol{v}}\right)\right](17)$

To distinguish between the behaviour of asphalt concrete in tension and compression, the spectral decomposition technique introduced by [Simo and Ju, 1987a], [Simo and Ju, 1987b] is used to decompose the stress and strain tensors into positive and negative components. The spectral decomposition technique is applicable to both the nominal and effective stress and strain tensors. Similar technique has been used by [Hansen and Schreyer, 1994], [Voyiadjis et al., 2009]. The positive part of the stress tensor can be extracted as:

$$
\boldsymbol{\sigma}^{+}=\sum_{i=1}^{3}<\sigma^{k}>\boldsymbol{n}^{\boldsymbol{k}} \otimes \boldsymbol{n}^{\boldsymbol{k}},
$$

where $\sigma^{k}$ are the eigenvalues and $\boldsymbol{n}^{\boldsymbol{k}}$ are the corresponding eigenvectors. Similar technique can be used to obtain the positive and negative components of the strain tensor. The negative part of the stress tensor can be obtained using the expression in Equation 19

$$
\sigma^{-}=\sigma-\sigma^{+}
$$

Once the positive and negative components of the stress and strain tensor have been obtained, the damage conjugate $(\boldsymbol{Y})$ can then be decomposed to obtain the positive $\left(\boldsymbol{Y}^{+}\right)$and negative $\left(\boldsymbol{Y}^{-}\right)$parts as well. $\left(\boldsymbol{Y}^{+}\right)$is the damage driving force under tensile conditions while $\left(\boldsymbol{Y}^{-}\right)$is the driving force under compressive conditions.

The prony series representation of the generalized Maxwell's model can be introduced and the undamaged (effective) relaxation function written in the form:

$$
\overline{\boldsymbol{E}}(\boldsymbol{t})=\overline{\boldsymbol{E}}_{\infty}+\sum_{i}^{n} \overline{\boldsymbol{E}}_{\boldsymbol{i}} \exp ^{-\frac{t}{\tau_{i}}},
$$

where $\tau_{i}$ is the ratio of the undamaged dashpot viscosity constant $\left(\bar{\eta}_{i}\right)$ and the elastic spring constant $\left(\bar{E}_{i}\right)$ of the $i^{\text {th }}$ dashpot component in the Maxwell model. The reduction of the stiffness due to damage is accounted on the long term $\boldsymbol{E}_{\infty}$ and the equilibrium stiffness $\boldsymbol{E}_{\boldsymbol{i}}$ and the damaged relaxation modulus can be expressed as:

$$
\boldsymbol{E}(\boldsymbol{t})=\boldsymbol{E}_{\infty}+\sum_{i}^{n} \overline{\boldsymbol{E}}_{\boldsymbol{i}} \exp ^{-\frac{t}{\tau_{i}}}
$$

To simplify the expression for the damage conjugate $(\boldsymbol{Y})$ in Equation 17, we take advantage of the prony series representation to re-write the damage conjugate in terms of the total effective stress $\overline{\boldsymbol{\sigma}}^{ \pm}$and the total viscoelastic strain as:

$$
\boldsymbol{Y}^{ \pm}=\frac{1}{2}\left[\boldsymbol{\epsilon}^{ \pm} \cdot \overline{\boldsymbol{\sigma}}^{ \pm}+\overline{\boldsymbol{\sigma}}^{ \pm} \cdot \boldsymbol{\epsilon}^{ \pm}\right]
$$

where $\overline{\boldsymbol{\sigma}}$ is the summation of the effective long term $\bar{\sigma}_{\infty}$ and equilibrium $\bar{\sigma}_{i}$ stress. 
4.1 Damage initiation and evolution

A non-associative damage formulation proposed by [Onifade et al., 2015] is used to derive different criteria for damage initiation and evolution. The initiation and evolution of damage is considered only on the positive part of damage conjugate $\boldsymbol{Y}^{+}$. The micro-crack initiation criteria is expressed in terms of the micro-crack initiation potential $\left(\wp_{1}^{*}\right)$, the critical micro-crack initiation threshold $\left(\wp_{1, c}^{*}\right)$, and the isotropic hardening parameter. The micro-crack initiation criterion in one principal damage axis is expressed as follows:

$$
f^{d}=\wp_{1}^{*}\left(\boldsymbol{Y}^{+}\right)-\wp_{1, c}^{*}\left(S_{o}\right)-R(r)=0,
$$

Where:

$\wp_{1}^{*}\left(\boldsymbol{Y}^{+}\right)$: is the micro-crack initiation potential

$\wp_{1, c}^{*}\left(S_{o}\right)$ : is the critical micro-crack initiation threshold

$\boldsymbol{Y}^{+}$: is a measure of the strain energy density

$S_{o}$ : is an energy term obtained from a strength test

The micro-crack initiation potential $\wp_{1}^{*}\left(\boldsymbol{Y}^{+}\right)$is driven by thermodynamic conjugate of the damage variable $\left(\boldsymbol{Y}^{+}\right)$and expressed as:

$$
\wp_{1}^{*}\left(\boldsymbol{Y}^{ \pm}\right)=\frac{S_{o}}{k_{2}+1} \cdot\left(\frac{\sqrt{\boldsymbol{Y}^{+}: \boldsymbol{Y}^{+}}}{2 \cdot S_{0}}\right)^{k_{2}+1}
$$

where $k_{2}$ and $S_{o}$ are material parameters. The critical micro-crack initiation threshold $\wp_{1, c}^{*}\left(S_{o}\right)$ is expressed in terms of $k_{2}$ and $S_{o}$ as:

$$
\wp_{1, c}^{*}\left(S_{o}\right)=\frac{S_{o}}{k_{2}+1}
$$

The micro-crack propagation criterion $F_{D}$ which is used to derive the evolution of damage is expressed as:

$$
F_{D}=\wp_{2}^{*}\left(\boldsymbol{Y}^{+}\right)-\alpha \cdot \wp_{1, c}^{*}\left(S_{o}\right)-R(r)=0,
$$

where $\alpha$ is $k_{1} / k_{2} \cdot \wp_{2}^{*}\left(\boldsymbol{Y}^{+}\right)$is the micro-crack propagation potential and expressed as:

$$
\wp_{2}^{*}\left(\boldsymbol{Y}^{+}\right)=\alpha \cdot \frac{S_{o}}{k_{2}+1} \cdot\left(\frac{\sqrt{\boldsymbol{Y}^{+}: \boldsymbol{Y}^{+}}}{2 \cdot S_{0}}\right)^{k_{2}+1}
$$

The evolution of micro-crack is obtained with respect to the dissipative micro-crack potential $F_{D}$ by taking the derivative of the dissipation potential. The resulting power-law type damage evolution law is given as:

$$
\dot{\boldsymbol{D}}=\lambda \cdot \frac{\partial \wp_{2}^{*}\left(\boldsymbol{Y}^{+}\right)}{\partial \boldsymbol{Y}^{+}}=\frac{k_{1}}{k_{2}} \cdot\left(\frac{\sqrt{\boldsymbol{Y}^{+}: \boldsymbol{Y}^{+}}}{2 \cdot S_{0}}\right)^{k_{2}} \cdot \frac{\boldsymbol{Y}^{+}}{\sqrt{\boldsymbol{Y}^{+}: \boldsymbol{Y}^{+}}} \cdot \dot{\lambda}
$$

where $\lambda$ is the Lagrange multiplier. From Equation 28, the material integrity parameter $\phi$ can be expressed as:

$$
\dot{\boldsymbol{\phi}}=\boldsymbol{I}-\frac{k_{1}}{k_{2}} \cdot\left(\frac{\sqrt{\boldsymbol{Y}^{+}: \boldsymbol{Y}^{+}}}{2 \cdot S_{0}}\right)^{k_{2}} \cdot \frac{\boldsymbol{Y}^{+}}{\sqrt{\boldsymbol{Y}^{+}: \boldsymbol{Y}^{+}}} \cdot \dot{\lambda}
$$


The Lagrange multiplier $(\lambda)$ is obtained by differentiating the micro-crack propagation criterion $\left(F_{D}\right)$ with respect to the hardening function $(R)$ and expressed as:

$$
\dot{r}=\dot{\lambda} \cdot \frac{\partial F_{D}}{\partial R}=\dot{\lambda}
$$

The material integrity parameter can then be expressed as:

$$
\dot{\boldsymbol{\phi}}=\boldsymbol{I}-\frac{k_{1}}{k_{2}} \cdot\left(\frac{\sqrt{\boldsymbol{Y}^{+}: \boldsymbol{Y}^{+}}}{2 \cdot S_{0}}\right)^{k_{2}} \cdot \frac{\boldsymbol{Y}^{+}}{\sqrt{\boldsymbol{Y}^{+}: \boldsymbol{Y}^{+}}} \cdot \dot{r}
$$

where, $k_{1}, k_{2}$ and $S_{o}$ are material parameters.

The viscoelastic damage model is used to estimate the accumulated damage in the specimen during the Superpave IDT strength test for determination of the the DCSE value from the test results.

\section{Materials and testing}

The Superpave IDT test results used for the re-interpretation of the strength test were obtained and re-analyzed from an earlier study on the evaluation of fracture and moisture damage performance of wax modified asphalt mixtures [Das et al., 2012a], [Das et al., 2012b]. Unmodified 70/100 penetration grade binder was used as the base binder and crushed granite aggregate type (AG2) is used for the fabrication of the asphalt concrete samples. The binder content of the mixture is $6.4 \%$ by weight and the target air-void was $7 \pm 1 \%$ by volume. Specimens of diameter $150 \mathrm{~mm}$ with a thickness of $100 \mathrm{~mm}$ were prepared using the Superpave gyratory compactor. The detailed fabrication process and the bitumen characteristic properties are presented in [Das et al., 2012b].

The resilient modulus and creep test results are used to obtain the viscoelastic properties of the mixture. The mixture strength and fracture properties are obtained from the Superpave IDT strength test. The experimental procedure proposed by [Roque and Buttlar, 1992] is used in the strength test for the identification of the point of macro-fracture initiation based on the comparative evaluation of the changes in the vertical and horizontal deformations in the center region of the test specimen. The mixture fracture properties are obtained at the observed instance of macro-crack initiation. The tests were carried out at a temperature of $-10^{0} \mathrm{C}$.

The results obtained from the Superpave resilient modulus test, creep test and IDT strength test for AG2 mixture are presented in Table 1 where $M r$ is the resilient modulus, $D 1$ and $m$ are power-law creep compliance parameters. $E E$, $D C S E$ and $F E$ are the elastic energy, dissipated creep strain energy and the fracture energy densities respectively.

Table 1: Summary of Superpave IDT test results for AG2 mixture

\begin{tabular}{clllllll}
\hline $\begin{array}{c}\text { Temp } \\
\left({ }^{\circ} C\right)\end{array}$ & $\begin{array}{l}\mathrm{Mr} \\
(\mathrm{Gpa})\end{array}$ & $\begin{array}{l}\mathrm{D} 1 \\
(1 / \mathrm{Gpa})\end{array}$ & $\mathrm{m}$ & $\mathrm{aT}$ & $\begin{array}{l}\mathrm{EE} \\
(\mathrm{kJ} / \mathrm{m} 3)\end{array}$ & $\begin{array}{l}\mathrm{DCSE} \\
(\mathrm{kJ} / \mathrm{m} 3)\end{array}$ & $\begin{array}{l}\mathrm{FE} \\
(\mathrm{kJ} / \mathrm{m} 3)\end{array}$ \\
\hline-10 & 17.09 & 0.057 & 0.761 & 0.0477 & 0.21 & 0.70 & 0.91 \\
\hline
\end{tabular}




\section{Finite Element Simulation of Superpave IDT test}

The viscoelastic damage model presented in Section 4 was implemented in a Finite Element program (COMSOL Multiphysics $($ ) ) for the identification of the damage parameters and simulation of the Superpave IDT strength test. Damage (in the form of micro-cracks) is considered as the source of deviation from the theoretical linear viscoelastic response at low temperature range and fast loading rate where plasticity is minimized. The theoretical response corresponds to the response of the material in the absence of damage. A three-dimensional (3D) FEM model is considered for the identification of the damage parameters to capture the effects of the three-dimensional stress distribution on the material response.

The default viscoelastic model in COMSOL Multiphysics $(\mathbb{B}$ assumes that the viscous part of the deformation is incompressible so that the volumetric deformation is purely elastic. For this reason, the default viscoelastic model is not used in the implementation of the damage model. A weak Partial Differential Equation (PDE) formulation is introduced in addition to the Linear elastic model to model the viscous behaviour that considers the viscous effect on both deviatoric and volumetric components.

The creep compliance response obtained from the creep test is used to obtain the viscoelastic behaviour of the asphalt concrete mixtures. A short-term response of $15 s$ is used in this study since the duration of the Superpave IDT strength test (up to the point of macro-fracture) is less than $2 s$ at the temperature considered. The prony series representation of the creep compliance is used to obtain the relaxation spectrum in the Laplace transform domain. The relaxation function obtained using the inverse Laplace Transform of the relaxation spectrum is represented with a generalized Maxwell model and used to model the viscoelastic behaviour of the material. Table 2 shows the viscoelastic material parameters used in the FEM model where $\mu$ is the total Poisson's ratio from the resilient modulus test, $E_{\infty}, E_{1}, E_{2}, \tau_{1}$ and $\tau_{2}$ are generalized Maxwell's model parameters.

Table 2: Viscoelastic material parameters

\begin{tabular}{cllllll}
\hline $\begin{array}{c}\text { Temp } \\
\left({ }^{o} C\right)\end{array}$ & $\begin{array}{l}E_{\infty} \\
(\mathrm{Gpa})\end{array}$ & $\begin{array}{l}E_{1} \\
(\mathrm{Gpa})\end{array}$ & $\begin{array}{l}E_{2} \\
(\mathrm{Gpa})\end{array}$ & $\begin{array}{l}\tau_{1} \\
(\mathrm{~s})\end{array}$ & $\begin{array}{l}\tau_{2} \\
(\mathrm{~s})\end{array}$ & $\mu$ \\
\hline-10 & 0.737 & 5.627 & 7.643 & 3.639 & 0.757 & 0.22 \\
\hline
\end{tabular}

\subsection{Identification of material damage parameters}

A three-dimensional (3D) Finite Element Method (FEM) model is used for the simulation of the Superpave IDT strength test for identification of the material damage parameters $S_{o}, k 1$ and $k 2$. A cylindrical geometry with a diameter of $150 \mathrm{~mm}$ and thickness of $50 \mathrm{~mm}$ is used in the FEM model. A steel loading strip of $20 \mathrm{~mm}$ is used in the Superpave IDT strength test experiment. Due to the symmetry of the test geometry, only one-eight of the entire geometry was modeled in the FEM model and the appropriate symmetry boundary conditions prescribed at the 


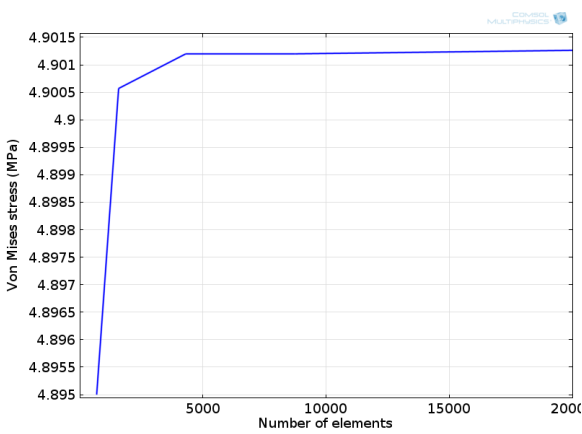

(a) Result of mesh convergence analysis

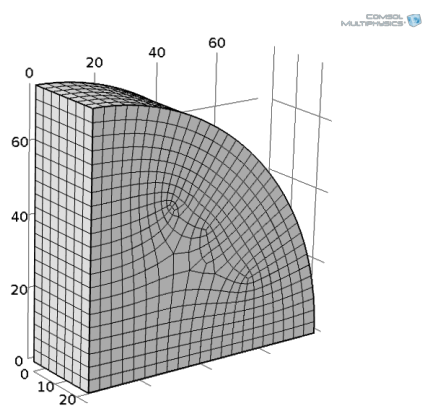

(b) Typical representation of the finite elements mesh

Fig. 2: Mesh convergence analysis and typical finite element mesh of the Superpave IDT geometry

planes of symmetry. The force $(P)$ exerted on the specimen during the Superpave IDT strength test was obtained and prescribed at the top of the FEM model geometry over half the width of the loading strip i.e. $10 \mathrm{~mm}$, to simulate the loading condition during the Superpave IDT strength test. The material parameters used in the FEM model is presented in Table 2.

A mesh convergence analysis was performed to determine the optimal number of FEM mesh elements for the model. The mesh convergence studies is carried with linear elastic material properties. Figure 2a shows the plot of the Von Mises stress with respect to the total number of mesh elements at the center of the specimen. Based on the result of the convergence analysis, a total of 4400 mesh elements are used for the Superpave IDT strength test simulation. Figure $2 \mathrm{~b}$ shows a representation of the mesh used in the FEM model.

Using the energy equivalence principle, the parameter $S_{o}$ corresponds to the value of the strain energy density at the instance of micro-damage initiation. The instance of micro-damage initiation is the point of deviation between theoretical and experimental strain energy densities, which is obtained through comparative evaluation of the rate of change of both energy densities.

After the identification of the damage parameter $S_{o}$, the viscoelastic damage model is activated and the COMSOL optimization module is used to minimize the point sum objective function in Equation 32. A point at the center of the geometry is selected for extraction of the strain values used in the objective function. The Nelder-Mead optimization method is used to solve the optimization problem to obtain the damage parameters $k 1$ and $k 2$. Table 3 shows the results of the identified damage parameters. Figure 3 shows a plot of the horizontal tensile strain at the center of the specimen for the nominal (damaged) configuration and the effective (undamaged) configuration. A relatively close match between the experimental strain observations and model response was obtained.

$$
\min \sum_{i=1}^{n}\left(\epsilon_{i}^{e x p}-\epsilon_{i}^{\text {model }}\right)^{2}
$$


where:

$\epsilon^{e x p}:$ is the horizontal strain at the center of the specimen from the Superpave IDT strength test

$\epsilon^{\text {model }}$ : is the horizontal strain at the center of the specimen obtained from the FEM damage model

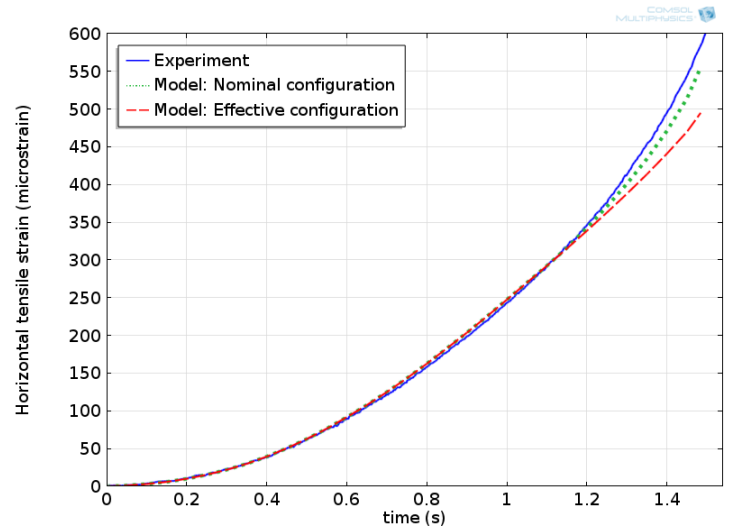

Fig. 3: Horizontal tensile strain in 11-direction: experiment, nominal (damaged) and effective (undamaged) configuration

Table 3: Damage model parameters for AG2 mixture

\begin{tabular}{clllc}
\hline Temp $\left({ }^{\circ} C\right)$ & $\mathrm{k} 1$ & $\mathrm{k} 2$ & So & Critical cracking threshold $(\mathrm{So} /(\mathrm{k} 2+1))$ \\
\hline-10 & 203 & 0.7 & 0.3 & 0.1765 \\
\hline
\end{tabular}

Figure 4a shows a contour plot of the typical stress distribution in the $\mathrm{x}$ direction obtained from the model i.e. $\sigma_{11}$. Very high compressive (-ve) stresses can be observed around the load application region which can result in local failure under the loading strip usually experienced in Superpave IDT strength tests particularly for thin specimens. The spectral decomposition technique can be used to decompose the components of the horizontal stress into positive and negative components. The positive component is used with the viscoelastic damage model for the damage estimation with respect to the observations in the Superpave IDT strength test. Figure $4 \mathrm{~b}$ shows a contour plot of the positive (tension) part of the stress in the x-direction $\sigma_{11}^{+}$.

Figure 5 shows a plot of the effective stress-strain response from the FEM model and the experimental response. There was a good match between the initial portion of the predicted effective and experimental response when the material response is in the linear viscoelastic range. The accuracy of prediction of the effective response was greatly increased when the shape and geometry of the test 


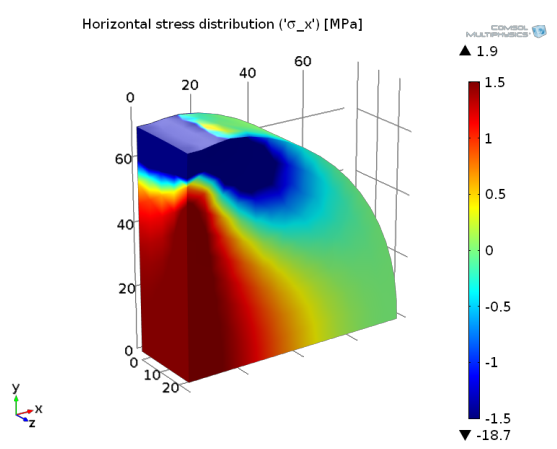

(a) Stress distribution in the 11-direction (horizontal)

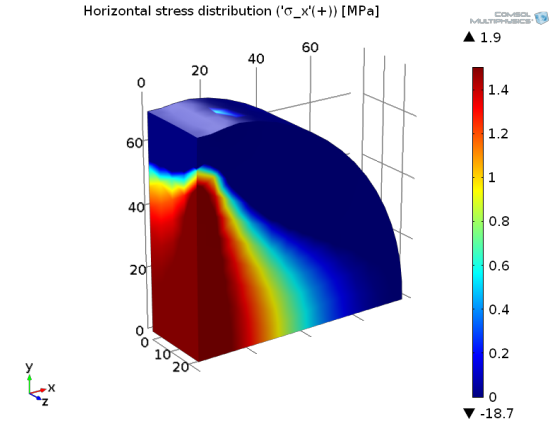

(b) Positive component of the stress tensor in the 11-direction (horizontal)

Fig. 4: Decomposition of stress tensor in the 11-direction (horizontal)

specimen is adequately represented. Assumption of a uni-axial condition at the center of the specimen results in an over-prediction of the effective response.

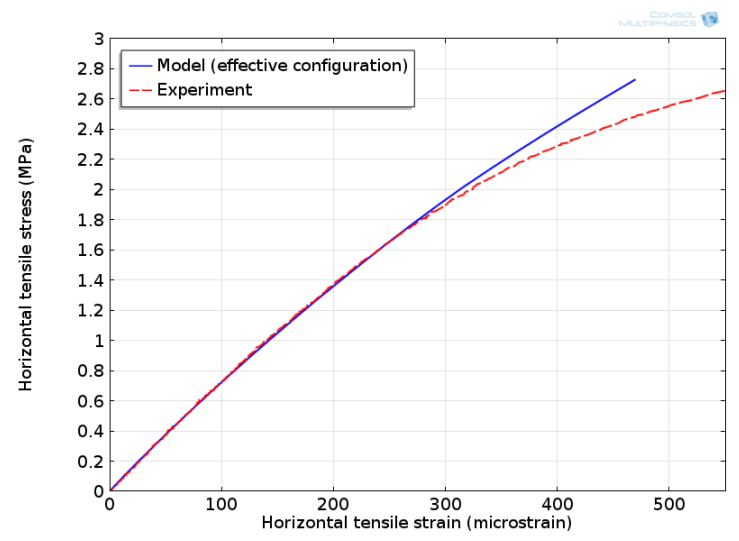

Fig. 5: Effective (undamaged) and experimental stress-strain response

Figure 6 shows the stress-strain response predicted using the viscoelastic damage model and the experimental response from the Superpave IDT strength test. The response are in relatively good agreement with the observed response in the experiment using the identified damage parameters. Comparative evaluation of the vertical and horizontal deformations at the gauge points was used to determine the instance of macro-crack initiation in the model for the extraction of the fracture properties.

Figures $7 \mathrm{a}$ and $7 \mathrm{~b}$ show the contour plots of the damage parameter $D_{11}$ and $D_{33}$ at the instance of the macro-crack formation. From Figure 7a, regions of high damage concentrations can be observed at a region about $25 \mathrm{~mm}$ from the top of 


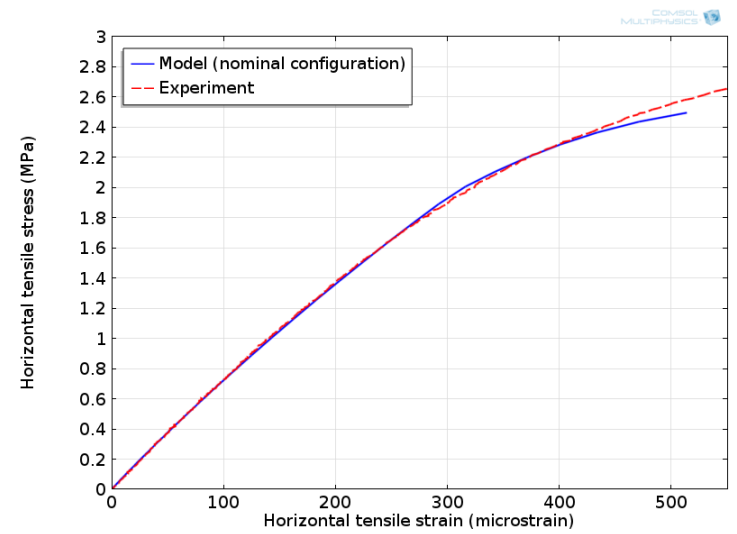

Fig. 6: Nominal (damaged) and experimental stress-strain response

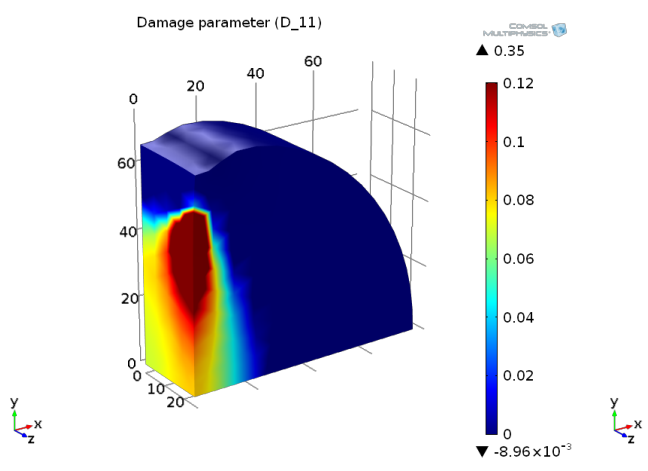

(a)

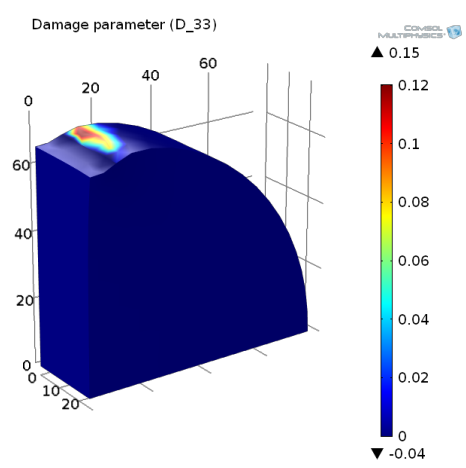

(b)

Fig. 7: Contour plot of damage variable (a) $D_{11}$ and (b) $D_{33}$ at $-10^{\circ} \mathrm{C}$

the specimen i.e. $1 / 3$ the radius of the specimen, which is outside the gauge length for the vertical LVDT.

To evaluate the influence of damage on the DCSE in the horizontal direction, the conventional approach and the recommended approach are used to obtain the DCSE from the FEM model response. The DCSE values are computed using the expressions for the conventional method $D C S E_{\text {conv }}$ and the recommended method $D C S E_{r e c}$ in Equations 33 and 34 respectively.

$$
\begin{gathered}
D C S E_{\text {conv }}=\frac{1}{2} \cdot \boldsymbol{\sigma}_{11}^{+} \cdot \epsilon_{11}^{+}-\frac{1}{2} \cdot \frac{\boldsymbol{\sigma}_{11}^{+} \cdot \boldsymbol{\sigma}_{11}^{+}}{\boldsymbol{E}_{\boldsymbol{o}}} \\
D C S E_{r e c}=\frac{1}{2} \cdot \boldsymbol{\sigma}_{\mathbf{1 1}}^{+} \cdot \boldsymbol{\epsilon}_{11}^{+}-\frac{1}{2} \cdot \frac{\boldsymbol{\sigma}_{11}^{+} \cdot \boldsymbol{\sigma}_{11}^{+}}{\boldsymbol{E}_{\boldsymbol{o}} \cdot\left(1-D_{11}\right)^{2}}
\end{gathered}
$$

where:

$D C S E_{c o n v}$ : is the DCSE value computed using the conventional approach 
$D C S E_{r e c}$ : is the DCSE value computed using the new recommended approach

Figure 8 shows the plot of the Fracture Energy (FE) density, the DCSE obtained using the conventional approach and the DCSE obtained using the recommended approach. The DCSE is a measure of material resistance to damage, it is expected that the resistance to damage decreases as the damage accumulates and evolves in the material. The recommended approach gives a better physical interpretation of the material response with regards to mechanisms of damage and fracture. On the other hand, the DCSE value obtained using the conventional approach does not give a good physical interpretation as it results in an increase in damage resistance with the evolution of damage as shown in Figure 8. Table 4 shows a summary of the results obtained from the FEM model analysis.

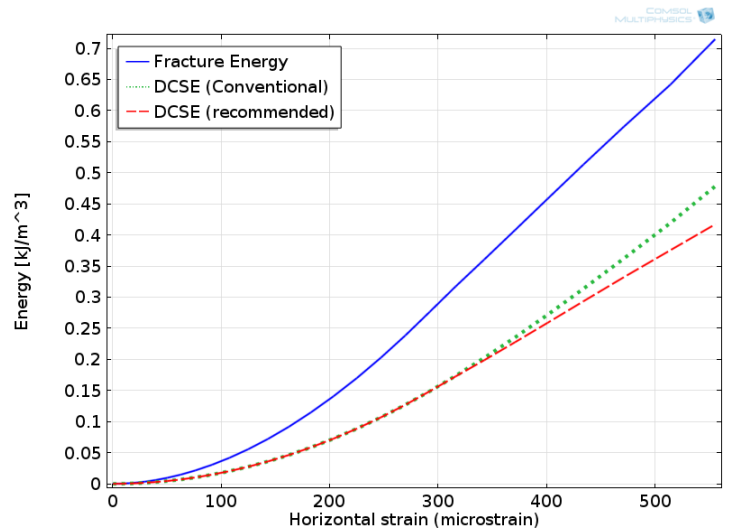

Fig. 8: Plot of fracture energy (FE) and dissipated creep strain energy (DCSE) in the 11-direction (horizontal) from FEM model.

Table 4: Summary of energy values deduced from the FEM model in the 11direction (horizontal)

\begin{tabular}{cllll}
\hline Temp $\left({ }^{\circ} C\right)$ & $\mathrm{FE}\left(k J / m^{3}\right)$ & $D C S E_{\text {conv }}\left(k J / m^{3}\right)$ & $D C S E_{r e c}\left(k J / m^{3}\right)$ & $D_{11}$ \\
\hline-10 & 0.68 & 0.41 & 0.37 & 0.082 \\
\hline
\end{tabular}

\section{Parametric Study}

A parametric study is performed to investigate the effect of varying deformation rates on the DCSE value obtained using the conventional approach and the recommended approach. The parametric study is performed using four different deformation rates $(0.5616 \mathrm{~mm} / \mathrm{s}, 0.2808 \mathrm{~mm} / \mathrm{s}, 0.1404 \mathrm{~mm} / \mathrm{s}, 0.0702 \mathrm{~mm} / \mathrm{s})$ prescribed at the top of the specimen. Figure 9 shows the effects of varying 
deformation-rates on the stress-strain response obtained from the damage model. Figures 10a shows the evolution of the strain energy density and Figure 10b shows the evolution of the damage parameter $D_{11}$ at different deformation rates. The viscoelastic damage mode captures the influence of the deformation-rate on the instance of damage initiation and the rate of damage accumulation. The model shows a lower rate of damage accumulation as the deformation rate is reduced as seen in Figure 10b. Comparative evaluation of the vertical and horizontal deformations at the points $P y$ and $P x$ respectively as shown in Figure 11a is used to identify the instance of macro-crack initiation. The instance of micro-crack initiation corresponds to the peak on the deformation difference curves at the different deformation rates as shown in Figure 11b.

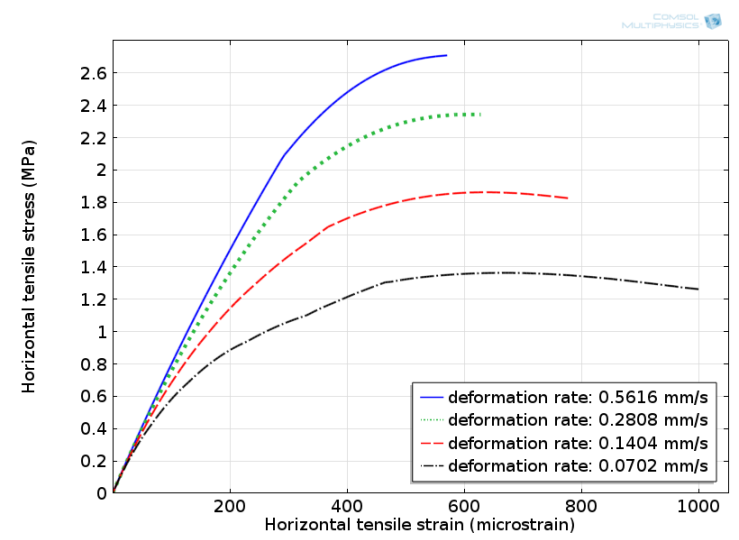

Fig. 9: Stress-strain response at different deformation rates

Figure 12a shows the plot of the critical damage parameter $D_{11}$ with respect to varying deformation rates. It can be observed from Figure 12a that the tendency to withstand induced damage accumulation before eventual macro-crack formation increases as the deformation rate decreases. Figure $12 \mathrm{~b}$ shows the plot of the DCSE values obtained using both conventional approach and recommended approach in Equation 33 and 34 respectively. It can be observed from Figure $12 \mathrm{~b}$ that the recommended approach captures the trend in the changes in the DCSE value as a function of deformation rate in a more consistent manner. It can also be observed that the error in the estimation of the DCSE values using the conventional approach is minimal at lower deformation rates, while the error estimate increases as the deformation rate increase. The results from the plot in Figure $12 \mathrm{~b}$ shows that fast loading rate (similar to rate currently used in the Superpave IDT strength test) reduces the effect of non-linearity on the material response as seen in Figure 9 (see the fastest loading rate). However, such fast loading rates doesn't necessarily guarantee an increased accuracy in the DCSE values determined as intended in the development of the test procedure. 


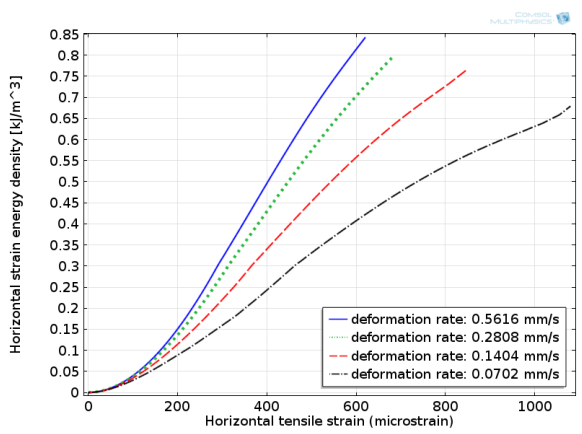

(a) Strain energy density at different deformation rates

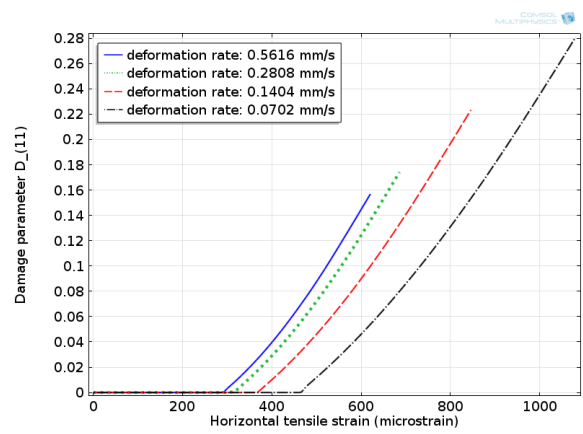

(b) Evolution of damage parameter $D_{11}$ at different deformation rates

Fig. 10: Evolution of strain energy density and damage parameter $D_{11}$ at different deformation rates

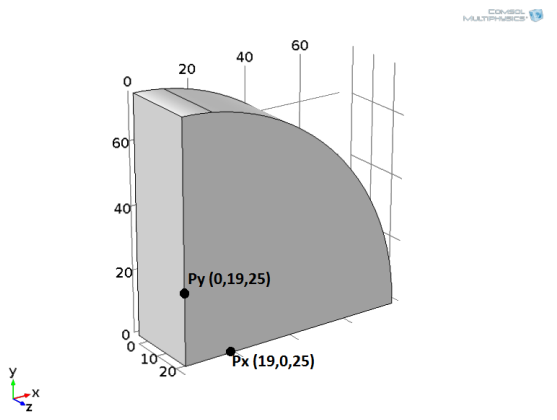

(a) Superpave IDT geometry showing points of vertical and horizontal strain evaluation

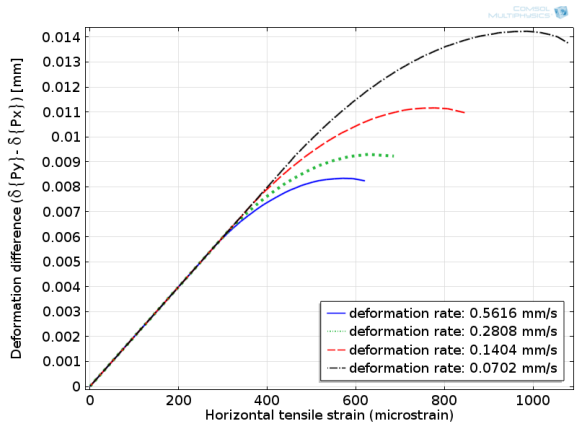

(b) Deformation difference between vertial deformation at Py and horizontal deformation at $\mathrm{Px}$

Fig. 11: Differential vertical and horizontal deformation evaluation for determination of point of macro-crack initiation

\section{Summary and Conclusions}

In this paper, a new method for the interpretation of the Superpave IDT strength test is presented. The method is based on a new viscoelastic damage formulation which is based on continuum damage mechanics and thermodynamics of irreversible processes. An anisotropic form of damage evolution is used to estimate the damage that accompanies the fracture process in the Superpave IDT strength test. The viscoelastic material behaviour and the accumulated damage are considered in the interpretation of the test results from the Superpave IDT strength test to obtain the energy-thresholds associated with macro-crack initiation in asphalt mixtures. 


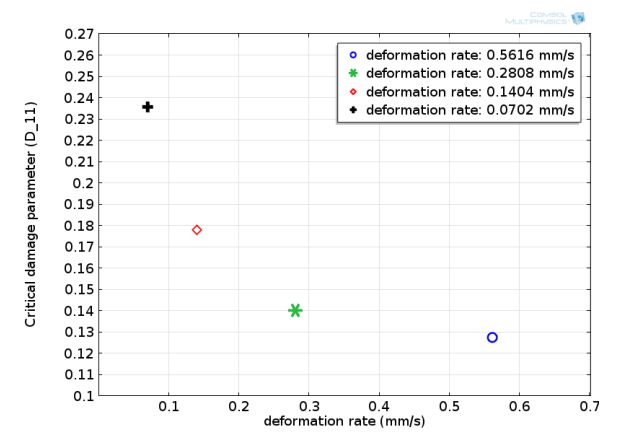

(a) Plot of critical damage parameter $D_{11}$ vs deformation rate

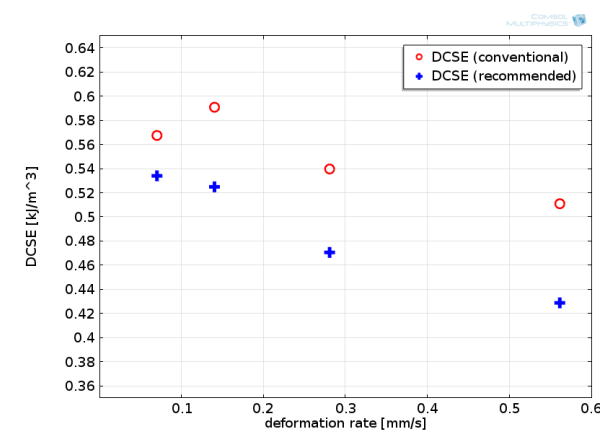

(b) Plot of DCSE vs deformation rate

Fig. 12: Plots of critical damage parameter $D_{11}$ and DCSE at different deformation rates

The results of this paper show that the introduction of an energy-based damage threshold within the framework of viscoelasticity appears to better capture the strain-rate effect which is important in the proper identification of fundamental energy limits such as FE and DCSE limits. Furthermore, the results presented in this paper also show that due to the complex bi-axial tension compression stress state at the center of the IDT specimen, the introduction of an anisotropic damage formulation further enhances the accuracy of the response prediction and better identification of the energy thresholds. The results also show that the recommended approach captures the trend in the changes in the DCSE value as a function of deformation rate in a more consistent manner. The error in the estimation of the DCSE value using the conventional method increases as the deformation rate increases.

Further work is needed for the examination of the existence of an intrinsic energy-based threshold for micro-crack initiation. Further work using different asphalt mixtures is needed to further validate the findings in this paper.

Acknowledgements The authors will like to acknowledge the support of the Swedish Transport Administration (Trafikverket) for funding this research.

\section{References}

[Abu Al-Rub and Voyiadjis, 2003] Abu Al-Rub, R. K. and Voyiadjis, G. Z. (2003). On the coupling of anisotropic damage and plasticity models for ductile materials. International Journal of Solids and Structures, 40(11):2611-2643.

[Balieu and Kringos, 2015] Balieu, R. and Kringos, N. (2015). A new thermodynamical framework for finite strain multiplicative elastoplasticity coupled to anisotropic damage. International Journal of Plasticity, 70:126-150.

[Birgisson et al., 2007] Birgisson, B., Wang, J., Roque, R., and Sangpetngam, B. (2007). Numerical implementation of a strain energy-based fracture model for HMA materials. Road Materials and Pavement Design, 8(1):7-45. 
[Carol et al., 2001] Carol, I., Rizzi, E., and Willam, K. (2001). On the formulation of anisotropic elastic degradation. i. theory based on a pseudo-logarithmic damage tensor rate. International Journal of Solids and Structures, 38(4):491-518.

[Christensen and Bonaquist, 2004] Christensen, D. W. and Bonaquist, R. F. (2004). Evaluation of indirect tensile test (IDT) procedures for low-temperature performance of hot mix asphalt. Number 530 in NCHRP report. Transportation Research Board, Washington, D.C.

[Cordebois and Sidoroff, 1982] Cordebois, J. P. and Sidoroff, F. (1982). Damage induced elastic anisotropy. In Boehler, J.-P., editor, Mechanical Behavior of Anisotropic Solids / Comportment Méchanique des Solides Anisotropes, pages 761-774. Springer Netherlands.

[Das et al., 2012a] Das, P. K., Tasdemir, Y., and Birgisson, B. (2012a). Evaluation of fracture and moisture damage performance of wax modified asphalt mixtures. Road Materials and Pavement Design, 13(1):142-155.

[Das et al., 2012b] Das, P. K., Tasdemir, Y., and Birgisson, B. (2012b). Low temperature cracking performance of WMA with the use of the superpave indirect tensile test. Construction and Building Materials, 30:643-649.

[Dinegdae et al., 2015] Dinegdae, Y. H., Onifade, I., Jelagin, D., and Birgisson, B. (2015). Mechanics-based top-down fatigue cracking initiation prediction framework for asphalt pavements. Road Materials and Pavement Design, 0(0):1-21.

[Hansen and Schreyer, 1994] Hansen, N. R. and Schreyer, H. L. (1994). A thermodynamically consistent framework for theories of elastoplasticity coupled with damage. International Journal of Solids and Structures, 31(3):359-389.

[Kim and Wen, 2002] Kim, Y. and Wen, H. (2002). Fracture energy from indirect tension testing. volume 71, pages 779-793.

[Koh, 2009] Koh, C. (2009). Tensile properties of open graded friction course (OFGC) mixture to evaluate top-down cracking performance. PhD thesis, UNIVERSITY OF FLORIDA.

[Lytton et al., 1993] Lytton, R. L., Uzan, J., Fernando, E. G., Roque, R., Hiltunen, D., and Stoffels, S. M. (1993). DEVELOPMENT AND VALIDATION OF PERFORMANCE PREDICTION MODELS AND SPECIFICATIONS FOR ASPHALT BINDERS AND PAVING MIXES.

[Nguyen et al., 2013] Nguyen, M. T., Lee, H. J., and Baek, J. (2013). Fatigue analysis of asphalt concrete under indirect tensile mode of loading using crack images. Journal of Testing and Evaluation, 41(1):104589.

[Onifade et al., 2015] Onifade, I., Birgisson, B., and Balieu, R. (2015). Energy-based damage and fracture framework for viscoelastic asphalt concrete. Engineering Fracture Mechanics, 145:67-85.

[Roque et al., 2004] Roque, R., Birgisson, B., Drakos, C., and Dietrich, B. (2004). DEVELOPMENT AND FIELD EVALUATION OF ENERGY-BASED CRITERIA FOR TOP-DOWN CRACKING PERFORMANCE OF HOT MIX ASPHALT (WITH DISCUSSION). In Journal of the Association of Asphalt Paving Technologists, volume 73.

[Roque et al., 2002a] Roque, R., Birgisson, B., Sangpetngam, B., and Zhang, Z. (2002a). CRACK GROWTH BEHAVIOR OF ASPHALT MIXTURES AND ITS RELATION TO LABORATORY AND FIELD PERFORMANCE.

[Roque et al., 2002b] Roque, R., Birgisson, B., Sangpetngam, B., and Zhang, Z. (2002b). HOT MIX ASPHALT FRACTURE MECHANICS: A FUNDAMENTAL CRACK GROWTH LAW FOR ASPHALT MIXTURES. In Journal of the Association of Asphalt Paving Technologists, volume 71.

[Roque and Buttlar, 1992] Roque, R. and Buttlar, W. G. (1992). THE DEVELOPMENT OF a MEASUREMENT AND ANALYSIS SYSTEM TO ACCURATELY DETERMINE ASPHALT CONCRETE PROPERTIES USING THE INDIRECT TENSILE MODE (WITH DISCUSSION). In Journal of the Association of Asphalt Paving Technologists, volume 61.

[Roque et al., 1999] Roque, R.. Zhang, Z., and Sankar, B. (1999). Determination of crack growth rate parameters of asphalt mixtures using the superpave IDT. Proceedings of the Association of Asphalt Paving Technologists, 68:404-433.

[Sangpetngam et al., 2003] Sangpetngam, B., Birgisson, B., and Roque, R. (2003). Development of an efficient hot mix asphalt fracture mechanics-based crack growth simulator. In Proc., 82nd Transportation Research Board Annual Meeting.

[Simo and Ju, 1987a] Simo, J. C. and Ju, J. W. (1987a). Strain- and stress-based continuum damage models-i. formulation. International Journal of Solids and Structures, 23(7):821840

[Simo and Ju, 1987b] Simo, J. C. and Ju, J. W. (1987b). Strain- and stress-based continuum damage models-II. computational aspects. International Journal of Solids and Structures, 23(7):841-869. 
[Voyiadjis et al., 2009] Voyiadjis, G. Z., Taqieddin, Z. N., and Kattan, P. I. (2009). Theoretical formulation of a coupled elastic - plastic anisotropic damage model for concrete using the strain energy equivalence concept. International Journal of Damage Mechanics, 18(7):603638.

[Zhang et al., 2001a] Zhang, Z., Roque, R., and Birgisson, B. (2001a). Evaluation of laboratory-measured crack growth rate for asphalt mixtures. Transportation Research Record: Journal of the Transportation Research Board, (1767):67-75.

[Zhang et al., 2001b] Zhang, Z., Roque, R., Birgisson, B., and Sangpetngam, B. (2001b). Identification and verification of a suitable crack growth law (with discussion). Journal of the Association of Asphalt Paving Technologists, 70. 